\title{
La antropología pedagógica en España durante el primer tercio del siglo $\mathrm{xx}^{1}$
}

\author{
CARMEN ORTIZ GARCÍA \\ Dpto. de Antropología. \\ CSIC. Madrid
}

\section{RESUMEN}

Partiendo de un concepto de la antropología como ciencia "moral y política", se repasan las relaciones y comunes intereses de la antropología y la pedagogía y se exponen algunas realizaciones concretas llevadas a cabo en España en el periodo anterior a la guerra civil, en relación con la antropología biológica, el folklore y la educación; especialmente las organizadas en la Escuela de Estudios Superiores del Magisterio de Madrid.

Palabras clave: Folklore, Educación, Antropometría, Antropología aplicada, Historia de la antropología.

\section{SUMMARY}

From the standpoint of a concept of anthropology as a "moral and political" science, the author reviews the relationships between, and common interests shared by, anthropology and pedagogy. She also discusses a number of specific achievements in Spain before the civil war in the fields of biological anthropology, folklore and education, especially those accomplished at the Escuela de Estudios Superiores del Magisterio in Madrid.

Key words: Folklore, Education, Anthropometrics, Applied Anthropology, History of Anthropology.

\section{LA ANTROPOLOGÍA, CIENCIA MORAL Y POLÍTICA}

Durante muchos años y en numerosas y variadas ocasiones, en clases universitarias, libros manuales o monografías específicas, se ha repetido hasta la

\footnotetext{
${ }^{1}$ La primera versión de este artículo fue una conferencia dentro del XXIII Curso de Etnología Española "Julio Caro Baroja". Antropología y educación: cultura y culturas en el aula, organizado por el Departamento de Antropología del CSIC y celebrado en Madrid entre el 24 y el 28 de marzo de 2003. Agradezco a su director, Luis Díaz G. Viana, la invitación y la oportunidad de participar en ese seminario.
}

RDTP, LVIII, 2 (2003): 71-92 
saciedad que la antropología era una disciplina exotista; una especie de sociología sencilla aplicada a los "primitivos", que no requería el uso de instrumentos tan sofisticados y tradicionales de los sociólogos como la estadística o las encuestas, porque se aplicaba a sociedades de pequeña escala, donde todo se podía llegar a conocer mediante las técnicas llamadas cualitativas, más profundas pero menos verificables; es decir, la entrevista y la observación directa de las conductas.

Puede que esto sea así; pero entonces resulta que nos encontramos con una gran cantidad de conocimientos, estudios y estudiosos que, a pesar de reclamar para sí la denominación de antropólogos y antropológicos, no se ajustan al retrato anterior, porque se ocupan de la propia sociedad (o de cualquier clase de sociedad) y emplean métodos de observación a veces más amplios que los de la observación directa —aunque no excluyan a ésta-, porque su objetivo es de tipo general. A algunos de estos conocimientos se les denomina con adjetivaciones añadidas (antropología médica, antropología de la educación, etc.), y también se les engloba bajo el nombre de antropología aplicada.

Está, pues, muy extendida la concepción de la antropología como la parcela de la sociología ocupada de las sociedades colonizadas por el Occidente, pero a la vez puede verse esa misma disciplina en función de su carácter eminentemente comparativo y, por tanto, como el estudio de las sociedades sometidas colonialmente; con más amplitud, como el estudio de la otredad $\multimap$ la diferencia social y cultural— en cualquier ámbito de dominación y espacio temporal en que ésta se produzca. Puede verse, así, la antropología como una disciplina que se ocupa tanto de nosotros como de otros y que emplea para su estudio herramientas y análisis propios que tienen que ver con la observación de conductas grupales y aprendizajes sociales y culturales con un nivel de detalle relativamente elevado. Si se quiere - mejor-, puede pensarse en la antropología como una disciplina que estudia comparativamente a las otras "culturas" para enseñarnos, así, más sobre la nuestra. En el campo de la antropología de la educación, ésta es una idea paradigmática, al menos desde la obra de Margaret Mead (Velasco, García y Díaz 1993: 13).

Aunque ésta pueda ser una postura minoritaria, no faltan en la disciplina quienes la aprecian como una ciencia "moral y política" y piensan que es un error histórico equipararla a un grupo particular de temas de estudio (Greenwood 1996: 387). De hecho, no sólo en Estado Unidos, también en otros países como Francia o Gran Bretaña, "la antropología se inició como una ciencia social amplia y un movimiento de reforma dirigida a eliminar los restos de la esclavitud, racismo, el genocidio de los indios americanos, y las cuotas de inmigración fijadas por los creyentes en la eugenesia" (ibid.). Y 
durante el primer tercio del siglo xx pueden encontrarse enorme cantidad de antropólogos y folkloristas que trabajan en ciudades y fábricas, y no en pueblos ni aldeas primitivas (ibid.).

En España hay ejemplos muy antiguos de esta antropología como ciencia moral, que se remontan a los siglos de oro y tratan sobre América, y hay también muchos casos de lo mismo en los siglos XIX y XX. Lo que resulta más difícil de encontrar es la sociología de primitivos o sencillos; excepto que consideremos como tal los estudios que tienen a España como "terreno", y a etnógrafos foráneos como autores.

\section{ANTROPOLOGÍA Y PEDAGOGÍA. RELACIONES E INTERESES COMUNES}

La segunda mitad del siglo XIX es una época de gran efervescencia en descubrimientos científicos y en la aplicación de éstos no sólo al conocimiento, sino también al cambio de la sociedad. Desde el darwinismo al marxismo, pasando por el organicismo funcionalista, encontramos en toda la ciencia positiva el mismo objetivo de conseguir, gracias a ella y sus avances aplicados al desarrollo social, una mejora definitiva de las condiciones de la existencia humana, en todos los sentidos. Es también éste el momento que puede considerarse como el de inicio de una comunidad científica dedicada a la antropología.

En líneas generales puede decirse que la antropología que se hace en España a partir del último cuarto del siglo XIX es una antropología de corte más naturalista que sociológica; es decir, que se interesa por "el hombre" en general, no por sus circunstancias concretas de vida; por su definición, tanto como ente espiritual como, sobre todo, en su parte de ser físico o biológico, distinto, aunque evidentemente en relación con otros seres de la naturaleza (Ortiz 1988a). Es una antropología que se ocupa del hombre en general o del hombre hispano en particular, no de otros hombres y culturas, aunque sobre éstos puedan también contarse ciertas obras y algún proyecto de interés. Otra característica que interesa remarcar es que el concepto de disciplina que se maneja no es el de la materia especializada que es usual hoy en día con el nombre de antropología social y cultural. Durante el siglo XIX, y en una buena parte también en el periodo anterior a la segunda guerra mundial, es relativamente común un concepto de la antropología como estudio de la humanidad y de la variedad de los grupos humanos, tanto sincrónica como diacrónicamente y tanto en las variaciones físicas como culturales (Ortiz 1996: 123). Puede, incluso, pensarse que la actual composición de la antropología por la antropología cultural, la biológica, la arqueología y la lingüística, usual en Estados Unidos, es una derivación de este concepto holístico 
existente en Europa en el siglo XIX y principios del xx, del que participaba, por ejemplo, Boas.

En cualquier caso, nos interesa para el tema tratado esta conceptualización de las ciencias antropológicas, porque incluye cuestiones sobre los orígenes de la cultura y la especie que, en última estancia, se ponían en relación con la infancia y, secundariamente, con la pedagogía. Se hablaba, así, de la "infancia de la humanidad" y de hecho muchos autores establecían un paralelismo más o menos directo entre mentalidad primitiva y mentalidad infantil; entre orígenes de la vida individual y de la vida colectiva, entre la simplicidad de las primeras fases del desarrollo de la persona y la sencillez técnica y de conocimientos de las poblaciones indígenas de muchas partes del mundo. El niño resultaba, así, una especie de "laboratorio" para experimentar con la mentalidad "infantil" que misioneros y administradores coloniales habían descrito como la propia de las comunidades aborígenes. Este puede ser considerado uno de los puntos de unión entre antropología y paidología y con la pedagogía. De hecho, el interés de muchos antropólogos por el campo educativo se basaba en la idea colonialista de la necesidad de "educar" las infantiles mentes y prácticas de los nativos administrados, por parte de sus metrópolis. En otro plano de dominación, la visión de la masa y el pueblo subdesarrollado como una infancia manejable por parte de las elites capitalistas ha sido explorada por los científicos sociales, como es bien conocido (ver por ejemplo Dorfman 2002: 13-14). De modo similar, el trabajo de algunos educadores — de forma sobresaliente las experiencias de Itard con el niño "selvático" del Aveyron (Itard 1982) o de otros investigadores con individuos privados de enculturación en su etapa infantil- interesó a muchos antropólogos por lo que tenían de "experimento" extrapolable a la reconstrucción de las fases iniciales de adquisición de la cultura. En relación a esto mismo, el estudio del atavismo evolutivo conectado con el de los anormales es otra perspectiva combinada que liga la teoría de la evolución humana y la capacidad de aprendizaje de la especie.

Pero había también otra fuerza que inclinaba el pensamiento sobre el hombre y la naturaleza humana hacia su configuración no física sino espiritual, y la posibilidad de su definición como ser trascendente, distinto a los otros seres de la naturaleza por sus posibilidades de elevación moral y espiritual. Indudablemente, la psicología fue la disciplina que más parcela ocupó en esta perspectiva; pero también intervino una antropología filosófica que pretendía obtener una definición del ser humano como una realidad trascendente y compleja, a la vez física y psíquica (Scheuerl 1985). Tal perspectiva no sólo existe en España en el momento que nos ocupa, sino que llega a ser incluso la predominante en el periodo de mitad del siglo XIX (Ronzón 1991) y tendrá, como veremos, interés por ser aplicada al mundo de la in- 
fancia y la educación. Por otra páıic, la visión holística de la persona y su apreciación psicológica obtendrán importantes resultados en este terreno, como la obra de Herman Nohl ([1938] 1965).

Antes de entrar a exponer con cierto detalle algunas realizaciones concretas que hubo en el campo de la antropología pedagógica en España a fines del siglo XIX y principios del $\mathrm{xx}$, me interesa todavía señalar otro punto de carácter general, o que se refiere más bien al contexto. Aunque la subdisciplina que normalmente se conoce como antropología de la educación es una cosa relativamente moderna y propia de la ciencia actual, en realidad desde mucho antes puede hablarse de un interés grande de la antropología hacia los procesos y las instituciones educativas, presente ya, por ejemplo, en Franz Boas que, de acuerdo con su formación dentro de la antropología naturalista europea, emprendió en Estados Unidos estudios sobre los índices de desarrollo de la población infantil y las necesidades de este desarrollo para llegar a ser óptimo en un entorno escolar (Castellote 1994: 71-73; García Castaño y Pulido 1994: 26). Los alumnos - mejor dicho, las alumnas - de Boas, Margaret Mead y Ruth Benedict fueron, en la siguiente generación, pioneras en los estudios comparativos de la educación infantil, pero introduciendo ya un giro en la metodología, que no insistía tanto en las condiciones de adaptación biológica de la población infantil, como en el desarrollo integral de la persona, en sus aspectos de carácter y personalidad, en un ámbito de cultura determinado (Mead 1972; Mead y Wolfenstein 1955).

Incluso en España puede considerarse que la antropología de la educación es una de las ramas fundadoras de la antropología profesional, ya que varias de las primeras tesis doctorales especializadas se dedicaron a la etnografía escolar (Díaz Maderuelo 1976; Caravantes 1985; García Castaño 1989; Díaz de Rada 1993; Alegret 1993).

Pero, hasta que se llega a esta definición moderna de una subdisciplina propia (Castellote 1994; Juliano 1996), puede hablarse de varias formas de relación entre antropología y educación (Ianni y Story 1976 en García Castaño y Pulido 1994: 38):

- Antropología en la educación: enseñanzas antropológicas en los curricula y programas de enseñanza; es la forma más tradicional de unir ambos campos.

- Antropología de la educación: la educación como campo significativo para la antropología en sí.

- Antropología y educación: unión de antropólogos interesados por la educación y educadores de orientación antropológica, tanto en lo conceptual como en lo metodológico. 
- Antropología aplicada a los problemas educativos: utiliza la teoría y los conocimientos de la antropología en las cuestiones sociales de relevancia educativa.

\section{ANTROPOMETRÍA ESCOLAR}

En el período acotado, encontramos en España algunos ejemplos y actuaciones que se podrían encuadrar en el primero y el último de los anteriores supuestos. A partir de la década de 1880 , empiezan a aparecer iniciativas que pretenden aplicar a la infancia y a las escuelas las nuevas técnicas de estudio y clasificación propuestas por la antropometría. El conocimiento de la morfología y la fisiología de los grupos humanos se consideraba, en la perspectiva naturalista que guiaba buena parte de la antropología decimonónica, como el objetivo primero y, según marcaban los conceptos de las ciencias positivas, el paso inicial para cualquier tipo de análisis o conclusión que se pudiera emprender posteriormente sobre la especie humana y sus variedades; conocimiento encaminado siempre - no hay que olvidarlo- en la ciencia positivista a conseguir la mejora de las condiciones sociales y de vida de los países. Así pues, no es de extrañar que una de las aplicaciones de este conocimiento se dirigiera a la educación y a la pedagogía, y que se estableciera que la antropología podía contribuir a sentar las bases de una pedagogía científica, apoyada en la realidad objetiva de las características psicofísicas de la infancia. Como parte de la renovación de la pedagogía y la práctica educativa, que en España tiene en la Institución Libre de Enseñanza su máximo exponente, se crean museos pedagógicos y laboratorios antropométricos específicos para recabar datos sobre las condiciones físicas de los niños y la incidencia que éstas puedan tener en su aprendizaje, con el fin de conseguir las mejores condiciones ambientales de todo tipo que contribuyan a mejorar el potencial físico-psíquico-social de las generaciones venideras.

Una de las bases de la moderna antropología era que se consideraba una ciencia inspirada y perteneciente al ramo de las ciencias naturales, capaz, por tanto, del mismo rigor y cientifismo, y provista de similares herramientas metodológicas y clasificatorias. Así pues, uno de los primeros recursos de la antropología pedagógica será la creación de instrumentos de medida y comparación, perfectamente establecidos y homologados. Las medidas y datos antropométricos —craneométricos fundamentalmente- eran en el siglo XIX el método en que se basaban las clasificaciones raciales; pero las mismas herramientas fueron también trasladadas a la criminología (no hay más que recordar las fichas y fotografías judiciales) y al ámbito educativo. La medición se percibía como la forma más exacta y fiable para obtener los caracteres 
regulares de grandes grupos de población. En el caso de la raciología, era la craneometría el estándar más utilizado, y así se elaboraban las llamadas "cranias" nacionales o por troncos raciales. En el caso de la antropología pedagógica los registros no eran exclusivamente craneométricos, sino que estaban más basados en las medidas en el vivo y en una antropometría más general, que consideraba las diferencias sexuales, raciales, los valores métricos (de la cabeza, el tronco y las extremidades), los caracteres fisiológicos (respiración, fuerza, expresión) y las proporciones corporales (García Castaño 1994: 96).

Se trataba de extender esta antropometría al mayor número posible de escolares para poder obtener conclusiones de tipo general, pero también se necesitaba que la recogida de los datos se hiciera de una forma fiable y estandarizada. Para ello, desde muy pronto se diseñan y aplican diferentes hojas y cartillas escolares, con el fin de obtener lo que Sergi denominaba la "carta biográfica de los alumnos". Una de las primeras fue la redactada en 1887 por Simarro, Salillas y Sáiz Campillo. Pero no sólo fueron médicos los que llevaron a cabo esta labor, y de hecho fueron los pedagogos -fundamentalmente el institucionista Manuel Bartolomé Cossío (Otero 1994)— los que pusieron en marcha gabinetes y museos, como el Museo Pedagógico Nacional de Madrid, donde existía un laboratorio de antropometría y de psicología experimental dedicado, según el propio director del Museo, "al estudio de las aplicaciones pedagógicas y a la propaganda de los métodos de la antropometría escolar" (citado en Puig-Samper y Galera 1983: 84). Este laboratorio, aunque modesto, estaba dotado con una serie de modernos aparatos (ibid.: 85).

También Rufino Blanco, aunque perteneciente a una tendencia educativa mucho más conservadora, manifestó un gran interés por esta clase de actividades. Guiado por el objetivo de instaurar una pedagogía científica —basada en las modernas técnicas de la psicología y la antropología que reclamaban un estudio científico del niño- tal como aparece plasmada en su obra Paidología y Paidotecnia. Pedagogía científica (1911), realizó él mismo contribuciones prácticas, entre las que pueden citarse el examen antropométrico de más de 6000 escolares madrileños (Puig-Samper y Galera 1983: 84), un curso de antropometría impartido en la Escuela Normal de Madrid en 1904 y, finalmente, su investigación empírica, Cómo crecen los niños españoles. Talla, busto y peso. Con algunos datos referentes a niños extranjeros, de 1920 (Molero y Del Pozo 1989: 151-154).

Pero las distintas hojas, registros y cuestionarios de antropología pedagógica que se ponen en marcha a partir de las dos últimas décadas del siglo XIX tienen un objetivo práctico, a la par que el del conocimiento de los caracteres morfológicos y fisiológicos de la población escolar, que es mejorar las condiciones físico-psíquicas (y también sociales, puede añadirse) de ese 
sector poblacional. Así, algunas de estas hojas — como la publicada en 1907 por el Museo Pedagógico Nacional, Las colonias escolares de vacaciones: hoja antropológica - estaban diseñadas para ser completadas antes y después de la asistencia de cada niño (obviamente de los que acudían a escuelas controladas por los pedagogos de la ILE o que confiaban en esta pedagogía científica) a las llamadas "colonias escolares de vacaciones". Se trataba de viajes vacacionales organizados escolarmente en los que se proporcionaba a los alumnos las condiciones óptimas (dentro de lo posible) de higiene y salubridad, alimentación, acondicionamiento de espacios de alojamiento y escolarización, ejercicio corporal, relaciones grupales, observación del entorno y aprovechamiento de la naturaleza, etc. Aunque no exclusivamente, ya que algunos profesores que trabajaban en el sistema de enseñanza público, en Escuelas, Institutos y Escuelas Normales, como Luis de Hoyos Sáinz, también las pusieran en práctica, estas colonias escolares fueron sobre todo organizadas por la Institución Libre de Enseñanza, a través, precisamente, del Museo Pedagógico Nacional. De hecho esta institución era la encargada de supervisar y procesar los datos antropométricos tomados a los escolares durante la colonia, que se publicaban luego en una memoria monográfica con los resultados de cada viaje (ver, por ejemplo, los diez volúmenes de Las colonias escolares... 1901-1924).

Sobre las primeras colonias de finales del siglo XIX se publicaron algunos estudios (Salcedo 1900; Las colonias escolares... 1898) y hay abundante información recogida en el órgano de difusión de la Institución, el Boletín de la Institución Libre de Enseñanza, en el que aparecen también artículos relacionados con la antropología pedagógica. Por ejemplo, la serie de J. de Caso, "La enseñanza de la antropología en la escuela", BILE, VII (1883), 152156; 187-190; 235-238; 285-287; VIII (1884), 266-268; IX (1885), 125-127; 134-137; de J. Fenton, "El folklore en relación con la psicología y la educación", BILE, VII (1883), 328-331; G. Flórez, "Un gabinete antropológico con aplicación a la pedagogía", BILE, XI (1887), 33-35; A. MacDonald, "Estudio antropológico y psico-físico de los niños de las escuelas de Washington", BILE, XXIII (1899), 98-102; y L. Simarro, "El exceso de trabajo mental en la enseñanza", BILE, XIII (1889), 37-39; 88-91; 369-373. (García Castaño 1994: 97).

\section{LA ANTROPOlOGÍA PEDAGÓGICA, UNA ANTROPOLOGÍA APLICADA}

En un nivel mayor de formulación, también encontramos tratados que intentan acoger los conocimientos propios y los objetivos de la nueva disciplina. Sin duda, el más conocido es el de Maria Montessori, Antropología pedagógica (1910), publicado en español en 1921, pero hay manuales bas- 
tantes años anteriores debidos a autores españoles. Así, en 1880, uno de nuestros más conocidos pedagogos, Pedro de Alcántara García publica unos Prolegómenos a la Antropología Pedagógica. De Gregorio Herráinz es el Tratado de Antropología y Pedagogía, de 1896, y en 1911 el Compendio de Antropología, Higiene escolar y Pedagogía de Pedro Díaz Muñoz iba por su quinta edición (la primera es de 1903). En el libro de Alcántara se define la nueva disciplina como:

La ciencia que, partiendo del conocimiento de la naturaleza humana en general, estudia la del niño en particular, sus manifestaciones principales y su desenvolvimiento hasta que el niño se convierte en hombre, todo ello con el fin de deducir consecuencias y hacer aplicaciones para la educación (1880: 43, citado en García Castaño 1994: 92).

En otra definición posterior aparece el mismo carácter:

\begin{abstract}
La más moderna, pero la más interesante y actual de las Antropologías aplicadas, es la Antropología pedagógica, nacida por la aplicación de los métodos al estudio del niño, y constituída en la actual crisis de la Pedagogía como base objetiva de la misma, al ser considerado el niño como una realidad concreta, no como una abstracción, ni como una reducción cuantitativa, pero no esencial, del hombre. Así nacida, ha englobado en su sentido amplio: el conocimiento de las formas y el crecimiento de las mismas, con los dos modos con que la Morfología humana y la Antropología concreta las estudian; el de las actividades y funciones fisiológicas, esencialmente en lo que al desarrollo y formación en las edades escolares atañe; y el del origen y evolución de los procesos psíquicos en ese período formador y plástico, cuyo conocimiento permite excitarlos y dirigirlos (Hoyos 1917: 30).
\end{abstract}

Como se ve, en realidad se trata de una antropología aplicada, que forma parte de una formulación más general que consideraba a la antropología (en su sentido biológico, más que en el cultural), como conocimiento científico de los grupos de poblaciones y, por tanto, como la base que debía tener en cuenta cualquier política social y económica que se pretendiese plantear en los distintos países y naciones. La antropología podía jugar este importante papel, debido a su situación intermedia entre las ciencias naturales y las sociales (Hoyos 1917: 3). Y, precisamente, a la parte aplicada de esta antropología con vocación social se la llamaba en España a fines del siglo XIX y principios del XX, antropología social. Alusiones a ella tenemos en la obra de Luis de Hoyos (Ortiz 1988: II, 628-629) y una explicación de contenidos aparece dada por Francisco María Tubino (1877), que emplea esa denominación para nombrar una nueva clase de estudios dirigidos al análisis de los diferentes grupos de un determinado territorio, mediante el conocimiento de sus orígenes, caracteres físicos, psicología colectiva, costumbres características, etc. con el fin de llegar a apreciar su idiosincrasia y poder, a 
partir de este conocimiento, dirigir las decisiones políticas y sociales que contribuyeran a unas mejores condiciones de la convivencia social (Sánchez Gómez 1994: 668-669).

Más conocidas por la historiografía - pero a mi juicio con idéntico sentido epistémico y además sin desarrollo, ya que quedaron únicamente en proyecto- son las lecciones de antropología programadas por Hermenegildo Giner para ser impartidas en la Institución Libre de Enseñanza. Me refiero a los titulados Programas de Biología y Antropología (1877) que, en un artículo pionero, fueron considerados por Carmelo Lisón (1977) como un hito precursor de la antropología social en España, anterior a la propia implantación de la disciplina en Inglaterra a principios del siglo xx. En la realidad, el concepto de antropología que muestra el programa de Giner es absolutamente filosófico y ,como tal, incluye desde la cosmología hasta la "antropología individual", que es la que contrapone a una última "antropología social". La tergiversación que hace Lisón de estos Programas (así como la reproducción de los mismos, que Lisón no incluye) queda detalladamente expuesta por Elena Ronzón (1991: 442-457), aunque no ha sido muy tenida en cuenta por los antropólogos.

También en los Congresos Pedagógicos, a partir del muy importante de 1882, y en los sucesivos planes de estudios de las Escuelas Normales aparecen alusiones a la necesidad del conocimiento y la enseñanza de la antropología pedagógica (García Castaño 1994), siempre en este sentido biológicopsicológico y aplicado. Pero me interesa ahora entrar en otras manifestaciones más o menos coetáneas de las que hemos visto hasta ahora y que inciden en otro de los modos de relación entre la antropología y la pedagogía que señalamos al principio. Me refiero a la utilización de materiales etnográficos en el curriculum escolar, y la investigación antropológica en la práctica educativa. En este caso, además, será el conocimiento de determinados rasgos culturales, y no de la antropometría o la morfología física, lo que se considere pertinente o de utilidad.

TRABAJOS ETNOGRÁFICOS EN LA ESCUELA DE ESTUDIOS SUPERIORES DEL MAGISTERIO

Además de las más conocidas instituciones creadas por los seguidores de Giner de los Ríos (Institución Libre de Enseñanza, Junta para Ampliación de Estudios, Residencia de Estudiantes, etc.), éstos tomaron iniciativas que, por distintas razones, se han resaltado menos por los historiadores. La Escuela de Estudios Superiores del Magisterio fue una de ellas. Fundada en 1909 por el impulso de Manuel Bartolomé Cossío, se trata de un precedente de la Facul- 
tad de Pedagogía, para cuya creación en la Universidad Central se disolvió, de hecho, la Escuela en 1932 (sobre la Escuela, ver Molero y Del Pozo 1989). En su nómina de profesores figuran personajes muy ilustres de la intelectualidad española, como Adolfo Álvarez Buylla, Luis de Zulueta, Rufino Blanco, Domingo Barnés, Concepción Sáiz, Ricardo Beltrán y Rózpide, etc. Entre ellos, figura un conocido antropólogo, Luis de Hoyos Sáinz, que fue profesor de Fisiología e Higiene escolar, pero desempeñó en la Escuela muchas otras funciones, entre ellas la de director (sobre Hoyos, ver Ortiz 1987).

La Escuela establecía en sus planes de estudio y sus técnicas docentes muchos elementos novedosos que tenían que ver con las modernas metodologías pedagógicas (Del Pozo 1989). Entre éstas, por ejemplo, destaca la introducción en el curriculum de nuevas asignaturas con este carácter, como psicología experimental, antropometría, psicometría, antropología pedagógica, fisiología e higiene escolar, pedagogía de anormales, etc. (ibid.: 70). Pero además, entre las novedades de trabajo en el aula, se incluía la elaboración de trabajos prácticos y la creación de laboratorios para la investigación; además de la realización de prácticas en centros externos. En estas tres iniciativas está presente la antropología.

Así, por ejemplo, en las clases de Hoyos se pasaba la Hoja Antropométrica creada por el mismo profesor, realizando los estudiantes entre sí las mediciones de estatura, talla, peso, perímetro, capacidad respiratoria, agudeza del campo visual, etc. y obteniendo después los índices y tablas correspondientes. Aparte de en los laboratorios propios de psicología experimental, fisiología y pedagogía de anormales, los alumnos llevaban a cabo experiencias de antropometría e higiene escolar, dirigidos por Hoyos, en centros públicos madrileños; pero, además, la escuela graduada "Príncipe de Asturias" se convirtió a partir de 1922 en centro anejo a la Escuela y en ella se creó un gabinete de antropometría y psicometría (ibid:: 74-76).

Sin embargo, donde se dieron plasmaciones más importantes fue en los Seminarios especiales, creados en la Escuela para que los alumnos desarrollaran trabajos de fin de carrera en materias conexas a las cursadas en su graduación. Tres de estos, ideados y mantenidos por Hoyos durante muchos años, tienen un contenido eminentemente antropológico: El Seminario de Etnografía, Folklore y Artes Populares, del que trataremos con algún detalle; el Seminario de Higiene Escolar, fundamentalmente práctico, que se dedicó sobre todo a la aplicación de la "Hoja de Inspección Higiénica de la escuela", del mismo Hoyos, en los colegios nacionales y a fomentar los contactos con los médicos escolares de la capital, encargados de la inspección de los centros de enseñanza (ibid:: 79); y el Seminario de Antropología Pedagógica y Paidología, coordinado por Hoyos, pero en el que también participaban Domingo Barnés, Rufino Blanco y Anselmo González. En éste, además de la 
utilización de las fichas antropométricas de Hoyos, Blanco y otros, a partir de 1928 se comenzaron a hacer memorias de fin de carrera con los datos obtenidos utilizando el "Cuestionario de primeros recuerdos" redactado por el propio Hoyos (ibid: 79-80), quien también participó en la dirección de algunos trabajos de psicopedagogía experimental (ibid.: 85). Durante todo el tiempo de funcionamiento de la Escuela existió una Comisión de Investigación, presidida por Hoyos, que aprobaba y supervisaba los temas y los métodos de los trabajos de investigación que los alumnos presentaban como memorias de graduación, primando criterios interdisciplinares y de rigor científico. Sobre todos los temas que hemos mencionado se realizaron numerosas memorias (Del Pozo 1989: 81-86; ver relación de memorias de fin de carrera en esta misma obra: 123-140).

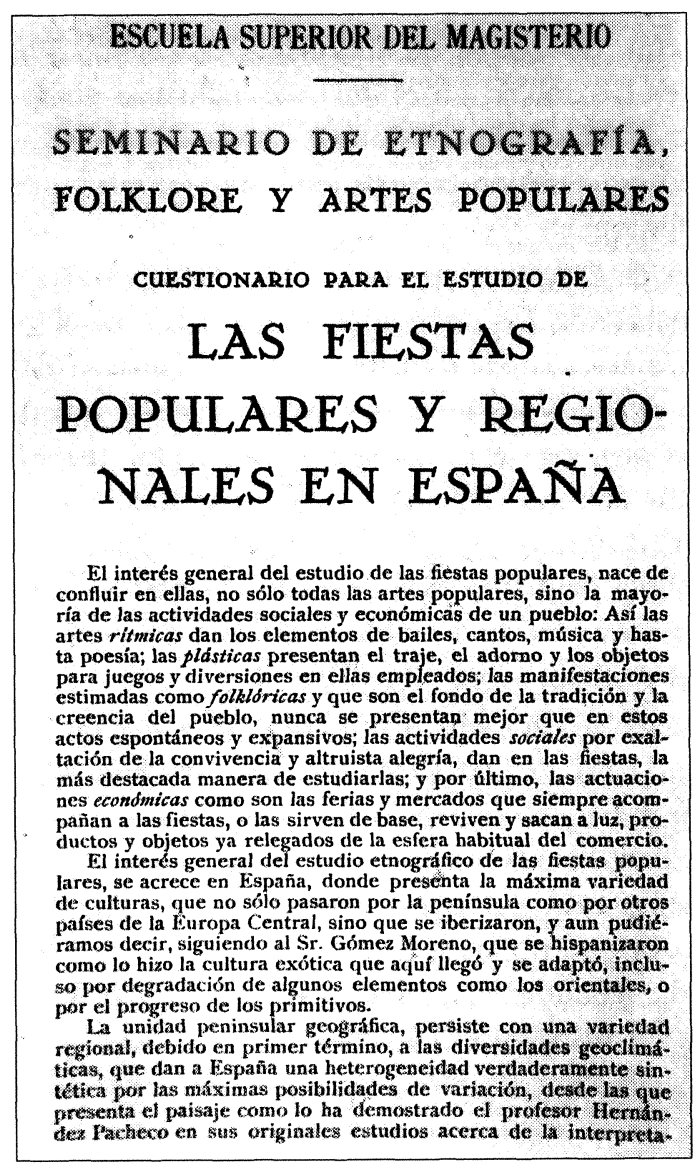

Figura 1.-Portada de uno de los cuestionarios redactados por Hoyos para su seminario de la Escuela.
Formando parte de esta enorme labor surgió en 1914 el Seminario de Etnografía, Folklore y Artes Populares, asimismo dirigido por Luis de Hoyos, que mantuvo gran actividad, produciendo cuestionarios, memorias y colecciones de objetos, durante todo el tiempo de funcionamiento de la Escuela y continuó luego, hasta 1936, en la Sección de Pedagogía de la Universidad Central, adonde Hoyos fue trasladado como catedrático (después de la guerra fue depurado y separado de su cátedra) (ver sobre esta iniciativa, Ortiz 1988b). Para el trabajo colectivo en el Seminario se marcaron una serie de líneas de investigación etnográfica, pensando en aquellos aspectos que corría más prisa documentar por el cambio tecnológico y económico que ya afectaba a la cultura tradicional de las zonas rurales y 
que, por otro lado, podrían resultar de recogida más sencilla y de mayor interés para los alumnos. Así, los temas de trabajo seleccionados fueron los medios de transporte, las fiestas, la alimentación, la casa y el ajuar doméstico, y las labores e indumentaria. De hecho, el traje folklórico fue el asunto a que más esfuerzo se dedicó.

A Hoyos le interesaba el traje porque pensaba que la conservación de grandes arcaísmos en la indumentaria popular de ciertas zonas ayudaba a aislar, mejor que otros elementos de la vida material más homogeneizados, el carácter particular de la cultura de determinadas regiones y, por ello, que la documentación de las formas de vestir, ya a punto de desaparecer, podía tener una gran importancia como elemento para el conocimiento de la diversidad antropológica de los pueblos de España. Así, se planteaba un programa de investigación general en el que sus alumnos irían recogiendo datos de la mayor cantidad posible de localidades que, luego, ellos emplearían en sus memorias de fin de carrera, pero que formarían un archivo de utilidad más amplia, en el que los datos sobre el traje se añadirían a otros sobre diversos aspectos, con el objetivo último - de tipo claramente positivistade llegar a dibujar el mapa de las áreas culturales existentes en nuestro país, que Hoyos, además, superponía al de las regiones previamente definidas en base a criterios antropométricos.

Lo que pretendía el Seminario era proveer, tanto a los alumnos de las Secciones de Letras y Ciencias como (y sobre todo; no hay que olvidar este aspecto en relación con el tema del traje) a los de Labores ${ }^{2}$, de un método que hiciera sistemática la recogida de la información, que debía obtenerse in situ con técnicas propias de la etnografía. Para ello, Hoyos impartía un cursillo sobre su propio concepto del "método etnográfico", y las profesoras de labores - Natividad de Diego y Luisa Díaz Recarte- daban una preparación especial sobre patrones, tipos de labores, terminología específica, etc. Finalmente, el profesor de historia del arte, Ángel Vegué, les introducía en lo referente a la historia del traje.

Con esta preparación, entre 1914 y 1931 se hicieron treinta y tres investigaciones particulares sobre el traje y las labores populares, la mayor parte centradas monográficamente en localidades y regiones concretas, que pueden ser desiguales en su valor (algunas llegaron a ser incluso publicadas), pero que aportaban un valioso material (escrito, fotografías y dibujos, patrones, muestras, etc.) que, aunque no con igual intensidad, cubría una buena parte del territorio nacional y cuyo acopio se realizó de una manera unifor-

${ }^{2}$ Entre cuyo profesorado se contaban Maravillas Segura - que había sido alumna en la promoción de 1920-, Natividad de Diego y África León; las tres autoras de libros especializados. 
me y minuciosa, marcada por el cuestionario redactado al efecto por Luis de Hoyos $(1922)^{3}$.

Cuando menos, las monografías proporcionaban datos descriptivos de todos los items especificados en ese cuestionario ${ }^{4}$ incluyendo además aspectos métricos y gráficos. La información había sido obtenida directamente en el terreno y luego complementada con un segundo grupo de información bibliográfica y documental, y con datos sobre el contexto de uso de los distintos trajes (ceremonias, fiestas, oficios, etc.). La utilización de los mismos criterios para la recogida de datos hacía que la información de cada caso tuviera cierta homogeneidad y, por tanto, fuera fácilmente clasificable y comparable; además, su minuciosidad aseguraba una base fiable para el análisis. El objetivo de Hoyos, de hecho, era configurar un archivo etnográfico y éste fue utilizado en varios de sus trabajos; pero además tuvo también plasmaciones colectivas importantes, como su presencia en la Exposición del Traje Regional de 1925 y en la etapa fundacional del Museo del Pueblo Español.

\footnotetext{
3 Ver, respecto al tema de las investigaciones y prácticas de los alumnos, así como la relación completa de memorias de fin de carrera presentadas en la Escuela, el trabajo de $M^{\mathrm{a}}$ del Mar del Pozo (1989: 65-140).

${ }^{4}$ Aparte de este cuestionario sobre el traje, Hoyos redactó varios otros para sus distintos seminarios de la Escuela de Estudios Superiores del Magisterio, la Facultad de Filosofía y Letras y el Museo del Pueblo Español: Cuestionario acerca de las regiones naturales de España. Madrid: Laboratorio de Metodología. Escuela Superior del Magisterio, 1914. Registros de antropometría escolar de veinte individuos. Madrid, 1914. Ficha del laboratorio de Antropología Fisiológica. Determinación de grupos sanguineos. Madrid, 1922. "Medios naturales o primitivos de transporte en las diversas regiones de España (Bases para un cuestionario)", Actas y Memorias de la Sociedad Española de Antropologí, Etnografía y Prehistoria, I, 1922, pp. 108-118. Cuestionario para el estudio de la alimentación popular regional de España. Madrid: Seminario de Etnografía, Folklore y Arte Popular de la Escuela Superior del Magisterio, 1924. Hoja de Antropología pedagógica. Madrid, 1926. Cuestionario para el estudio de las fiestas populares y regionales en España. Madrid: Seminario de Etnografía, Folklore y Arte Popular de la Escuela Superior del Magisterio, s.f. [1926]. Cuestionario acerca de los primeros recuerdos (Investigación del origen de la memoria en el niño). Madrid: Escuela Superior del Magisterio, 1928. Hoja para el estudio de la berencia en el bombre.- Grupos sanguíneos y caracteres antropológicos. Madrid: Laboratorio de Antropología fisiológica, 1929. Ficha de inspección higiénica de las escuelas. Madrid, 1931. Hoja de inspección médico-escolar. Madrid: Laboratorio de Higiene de la Facultad de Filosofía y Letras, 1933. "Cuestionarios especiales de Etnografía y Folklore", Anales del Museo del Pueblo Español, I, 1935, pp. 59-64. "Ficha antropológica para la investigación de la herencia", IV Congreso Internazionale di Patologia Comparata. Roma, 1939, II, pp. 321-326.
} 


\section{LA EXPOSICIÓN DEL TRAJE REGIONAL}

Al menos desde 1915, y junto con Telesforo de Aranzadi, había insistido Hoyos ante varios órganos de la Administración sobre la necesidad y urgencia de crear un Museo Etnográfico Nacional. Aunque con menos ambición, en su Seminario de la Escuela, Hoyos había conseguido reunir una pequeña colección de objetos, prendas, patrones y un abundante material gráfico, formado por 462 fotografías y 162 acuarelas tomadas del natural. En 1923 se estaba preparando una exposición sobre el traje regional español, patrocinada por la Real Academia de San Fernando y una junta de aristocráticas personas (Conde de Romanones, Duquesa de Parcent, etc). La circunstancia de morir prematuramente el director de la muestra, hizo que Hoyos fuera nombrado director en 1924, en función de los trabajos llevados a cabo en este campo.

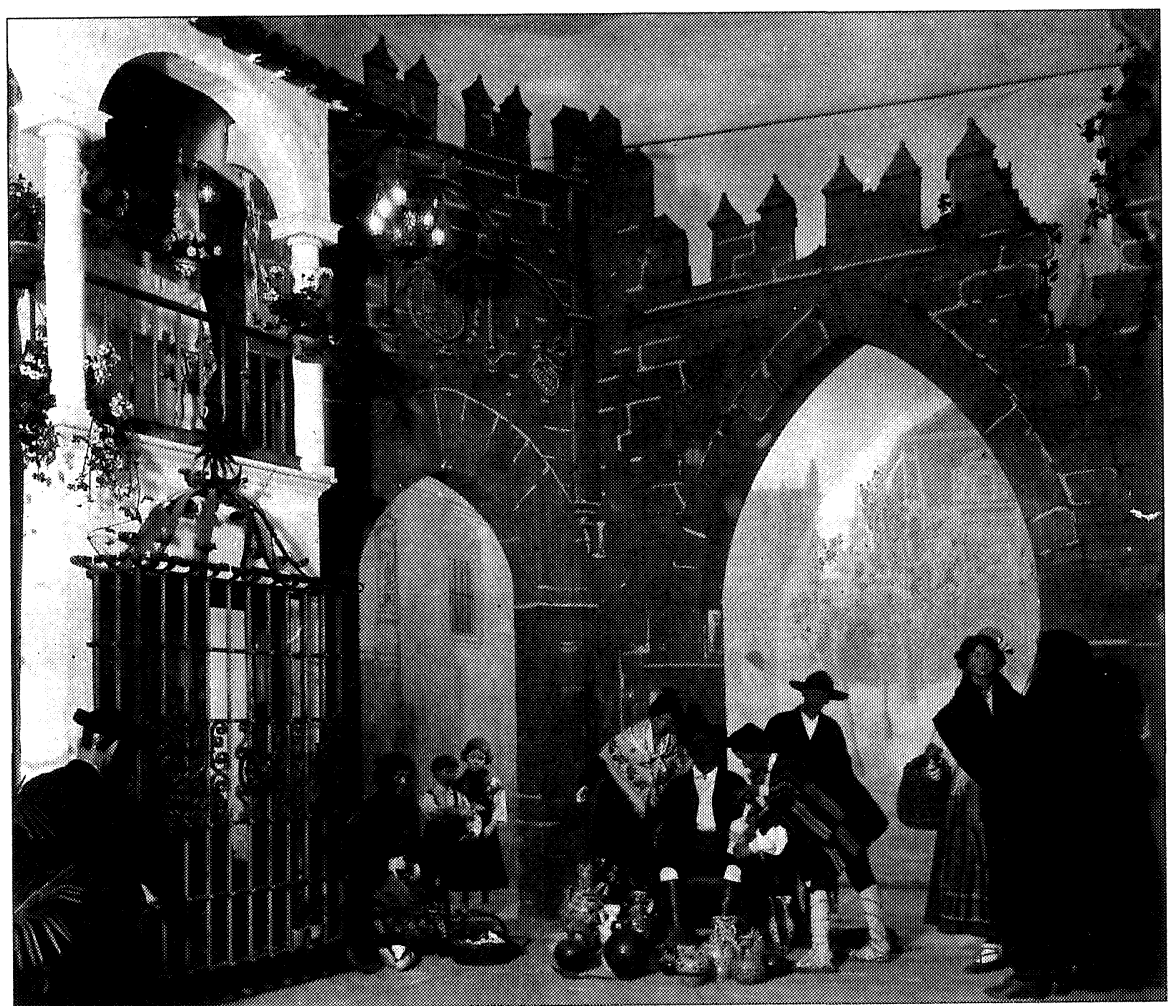

Figura 2.-Una de las "escenas" montadas en la exposición de 1925. La Puerta del Pópulo de Jaén. 


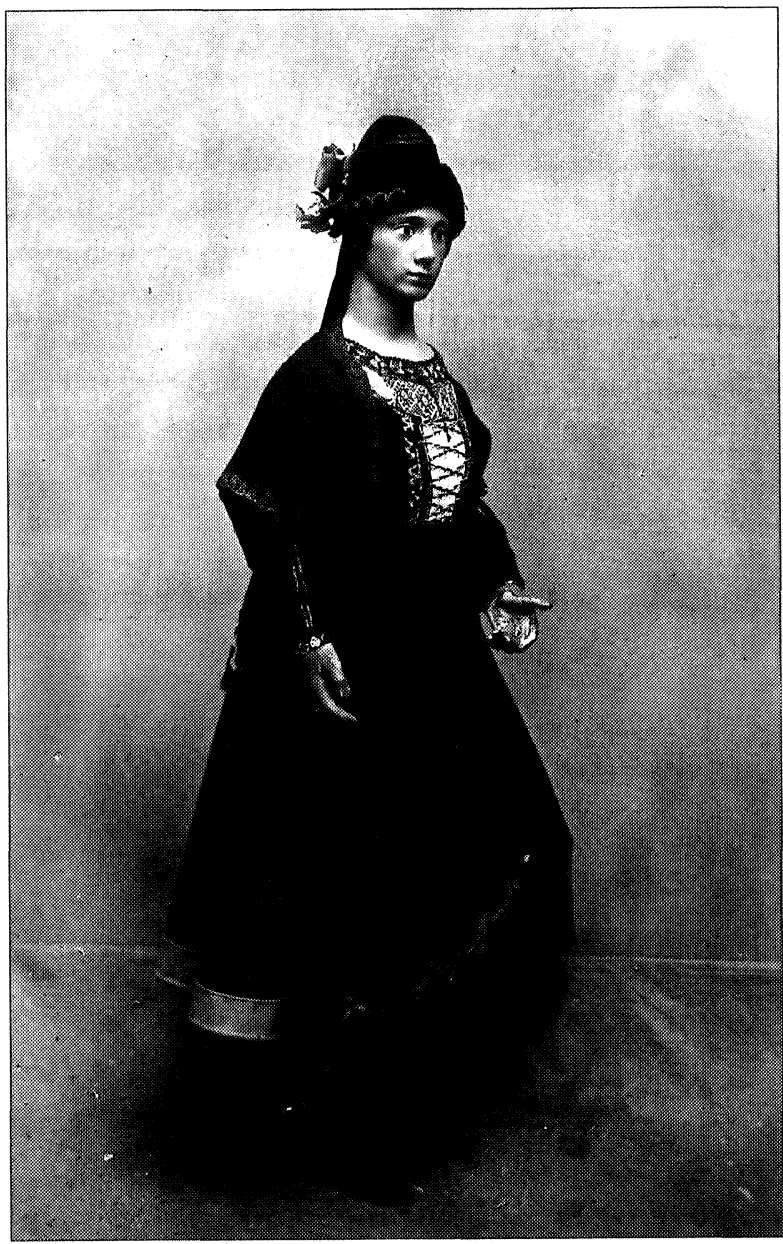

Figura 3.-Maniquí vistiendo el traje femenino de El Barranco (Ávila) en la exposición de 1925.
La exposición, que se inauguró en la $\mathrm{Bi}$ blioteca Nacional de Madrid el 18 de abril de 1925, estaba compuesta por una primera parte con una serie de escenas reproduciendo hogares y lugares tradicionales, y cuadros de costumbres. A esta sección de "escenas" seguía el "Salón de Trajes Regionales", en el que se situaron los trajes vestidos por maniquíes (agrupados en diez regiones). Además, en armarios, numerados de la A a la L, se exhibieron los distintos elementos del vestido, clasificados en grupos de prendas similares pertenecientes a cada región, para permitir la comparación tipológica. Esta segunda parte estaba acompañada por material gráfico y, en vitrinas y estantes especiales, se podían ver joyas y diversos objetos de oficios, ajuar, culto, etc. Los trajes de época formaban otra sección. Pero, además, a continuación de los armarios donde estaban las prendas sueltas, en el catálogo de la exposición (no gráfico) (Exposición 1925), se señala la ubicación de la "Instalación de la Escuela Superior del Magisterio. Seminario de Etnografía, Arte y Labores". En efecto, en el patio se colocó una vitrina con treinta y tres memorias monográficas sobre trajes de distintas provincias, realizadas por alumnas de las promociones de 1915 a 1925; se expusieron también fotografías y dibujos, papeletas bibliográficas y papeletas metódicas de trajes y prendas, en orden geográfico y tipológico (Ortiz 1988b: 452). 


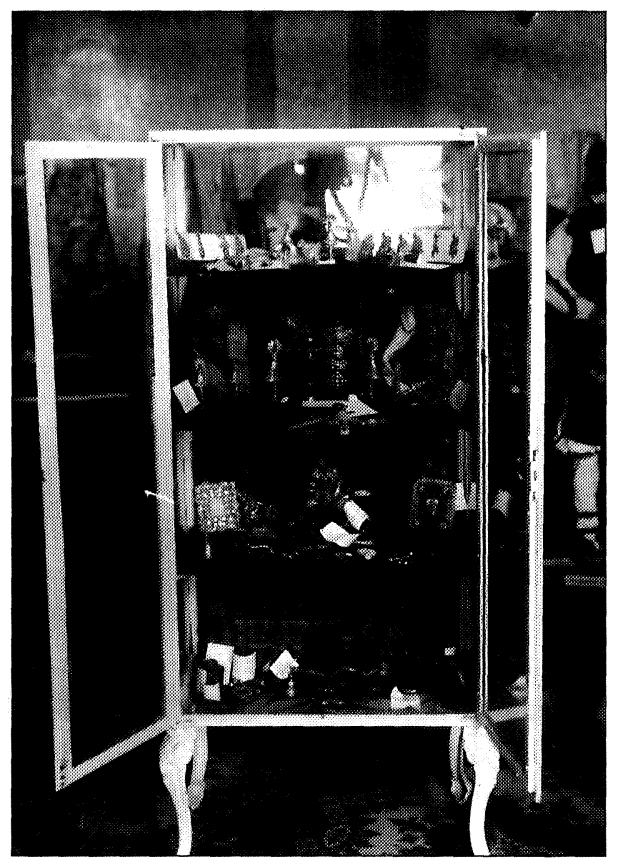

FIGURA 4.-Vitrina de la exposición de 1925 , en la que pueden apreciarse numerosos objetos asociados con la indumentaria: religiosos (cruces, vírgenes del Pilar, relicarios), supersticiosos (hilos con dijes infantiles) y puramente ornamentales (pendientes y aderezos).
El éxito que consiguió la Exposición de 1925 dio a conocer el Seminario y animó a su director a seguir difundiendo su labor. Así, en el I Congrès International des Arts Populaires, celebrado en Praga del 7 al 13 de octubre de 1928, consiguió reunir una buena cantidad de comunicaciones para la representación oficial de España en el Congreso, procedentes de folkloristas catalanes y de todo el país, pero sobre todo de sus colaboradores y alumnos de la Escuela del Magisterio. En total se presentaron en ese congreso sesenta y una comunicaciones sobre España, que fue, así, la delegación que más contribuciones presentó (los resultados del congreso se publicaron en dos volúmenes titulados, Art Populaire 1931).

La Escuela Superior de Magisterio desaparece en 1931, pero esto no supone la finalización del Seminario. En 1933 se recrea, de hecho, el Seminario de Etnografía y Folklore en la Sección de Pedagogía de la Facultad de Letras de la Universidad Central de Madrid.

Por otro lado, como ya se ha comentado, Hoyos siempre pensó que el Seminario podría ser el germen de un futuro museo etnográfico; la Exposición de 1925 consolidó esta idea y, como resultado de ese evento, se fundó el Museo del Traje Regional e Histórico. Sin embargo, Hoyos tenía una idea distinta: la indumentaria era un elemento cultural más de los que debían estar representados, entre otros muchos, en un museo etnográfico. Finalmente, su criterio se impuso y en 1934 el Museo del Traje quedó integrado en el Museo del Pueblo Español —de nueva creación - del que pasaron a formar parte las colecciones y documentos (escritos y gráficos) acumulados en la Escuela del Magisterio. Además, Hoyos aprovechó para vincular al museo, del que fue nombrado director, a muchos de sus alumnos y colaboradores: Angel Vegué - profesor de arte en la Escuela - será el subdirector, y sus alumnas Nieves de Hoyos, Francisca Vela, Máxima Oliver y Francisca Martínez Meléndez, serán 
nombradas auxiliares técnicas. Finalmente, otros ex-alumnos se incorporarán a la organización del museo a través de las figuras de los patronos regionales y correspondientes provinciales (Ortiz 1988b: 454) y, desde estas posiciones, colaborarán con su antiguo profesor, respondiendo a sus cuestionarios y gestionando la adquisición de fondos e informes desde las localidades en que estaban destinados como profesores normales o inspectores de primera enseñanza.

\section{EL FOLKLORE Y LA ESCUELA FRANQUISTA}

La guerra civil tuvo un efecto devastador en la vida del Museo y también en la de algunos de sus trabajadores. El régimen franquista depuró además de una forma sistemática al profesorado en todos sus niveles, desde el básico al universitario, y varios de los alumnos de la Escuela que habían tenido responsabilidades de gobierno durante la República - Rodolfo Llopis o Juan Comas, por ejemplo- fueron al exilio; otros, como Vicente Risco, mantuvieron una relación dramática con el falangismo y la mayor parte no tuvo más salida que buscar la supervivencia interior. Sin embargo, al régimen salido de la guerra le interesaba el folklore tanto o más de lo que había interesado a los nacionalismos periféricos en la etapa anterior. Y, además, la escuela era uno de los lugares donde el control de las ideas y los conocimientos sobre el "pueblo" y la cultura popular podía ser mayor y más sistemático.

Así pues, durante todo el franquismo el folklore fue una materia, si no curricular, al menos sí contemplada en la práctica docente, y dentro de la cual se llevaron a cabo también algunos intentos de investigación. Aunque ya fuera del ámbito temporal acotado para este trabajo, la obra de folkloristas como Bonifacio Gil (1964a y 1964b) y Arcadio de Larrea son paradigmáticas; incluso de este último podemos contar con un "manual" de uso para la utilización del folklore en el aula, que resulta muy interesante: El folklore y la escuela. Ensayo de una didáctica folklórica (1958). Ya en 1936 el folklorista y musicólogo Eduardo Martínez Torner (cuyo hermano Florentino fue también alumno de la Escuela de Estudios Superiores del Magisterio) había publicado un libro dedicado al empleo de la música folklórica como recurso pedagógico, pero lo que se propone Larrea es algo diferente; de hecho, él mismo menciona la diferencia de objetivos entre su libro y el de Torner (Larrea 1958: 13). Lo que se pretende ahora es poner al alcance del maestro, especialmente el que ejerce en el medio rural, las herramientas conceptuales y metodológicas necesarias para que conozca y estudie el folklore de la localidad en la que vive. 
En primer lugar, se trata de que ese conocimiento facilite la tarea docente en el aula, ya que para la enseñanza de cualquier materia puede encontrarse material útil en la cultura popular: "[...] religión, lenguaje, medicina, arte, técnica; en fin: toda suerte de conocimientos hallarán en la tradición popular acervo donde proveerse con abundancia y provecho" (Larrea 1958: 11). No se trata así de que el folklore se considere una asignatura más, sino que se propone como un elemento fundamental en la enseñanza de cualquier materia; en el sentido de que provee del conocimiento "popular" correspondiente en cada parcela de la enseñanza "culta" o reglada por los contenidos escolares (ibid.: 66). Una distinta utilidad, que señala Víctor García Hoz en el prólogo de la obra de Larrea (1958: XII), está bastante en consonancia con la función que otras instancias del régimen franquista otorgaba al folklore:

[...] podemos pensar finalmente que a través del folklore, cultivando la alegría de la vida popular, se puede contribuir a la solución de ese gran problema de la tristeza de nuestra vida rural. Magnífica tarea la de aquella escuela que logre alegrar la vida campesina y afincar con ilusión y con cariño a los hombres del pueblo en su pueblo mismo.

Con todo, a quien más puede beneficiar el conocimiento del folklore no es precisamente al "pueblo", sino al propio maestro, ya que éste vive y trabaja en un ambiente con el que necesariamente tiene que interaccionar para conseguir su objetivo, que Larrea muestra — no sé si conscientemente- que no es otro que "enculturar" a la población campesina; es decir sustituir su cultura popular y tradicional por otra cultura, culta y moderna (1958: 11). Señala como un hecho curioso que los campesinos "estimen en más el conocimiento del saber popular que el del saber culto, acaso porque el último les parezca de más fácil adquisición" (ibid.: 13), y refiriéndose al tópico de la "soledad" e incomprensión del maestro rural, opina que una de las causas de este aislamiento procede de la falta de interés recíproco entre el maestro y la sociedad en que vive. Larrea retrata la figura del maestro como un extraño cuyo bagaje cultural no es del interés del pueblo porque cumple funciones menos "prácticas" o útiles para la comunidad que las que desempeñan otros oficiantes externos (la salud física y espiritual, por ejemplo, que se ponen más indiscutiblemente en manos del médico o el sacerdote):

El Sacerdote y el Médico, por la índole missma de su misión, están directa e inmediatamente interesados en los problemas vivos y actuales del pueblo; no así el Maestro, cuya acción se proyecta más bien hacia el futuro y, en gran parte, hacia una zona que el pueblo considera superflua, dado el concepto estrictamente utilitario [sic] que el pueblo confiere a casi todas, si no a todas las manifestaciones de su vida y de su cultura (ibid.: 13). 
Lo que se plantea en esta visión de la relación entre el maestro y su pueblo no es otra cosa que la distancia enorme que puede haber entre la enseñanza y la cultura; entre la variedad real de los comportamientos culturales y la necesidad de homogeneizar y normativizar a los individuos que tiene la institución escolar. Esta tensión es la que parece subyacer en cualquier relación que se establezca entre las dos disciplinas — antropología y pedagogía - y en su mediación seguramente el folklore, su conocimiento y su enseñanza en la escuela, siempre que se haga de una manera rigurosa, puede tener un papel de los más importantes.

\section{BIBLIOGRAFÍA UTILIZADA}

AlCántara García, Pedro De. 1880. Prolegómenos a la Antropología Pedagógica. Madrid: English y Gas.

Alegret, JuAn L. 1993. Cómo se enseñan los otros. Análisis de la presentación racialista de la diversidad étnica en los libros de texto de EGB, BUP y FP utilizados en Cataluña en la década de los 80 . Tesis doctoral. Universidad Autónoma de Barcelona.

Art Populaire. 1931. Travaux artistiques et scientifiques du 1er Congrés International des arts populaires. París: Editions Duchartre, 2 vols.

Caravantes García, Carlos M. 1985. Antropología y procesos de endoculturación. Modelo etnográfico para el análisis de una institución educativa. Tesis doctoral. Universidad Complutense de Madrid.

Castellote Herrero, Eulalia. 1994. "Antropología y Pedagogía". Revista de Dialectología $y$ Tradiciones Populares XIIX (2): 69-79.

Colonias escolares de vacaciones (1891-1898), Las. Museo Pedagógico Nacional. 1898. Madrid: Fortanet.

Colonias escolares de vacaciones, Las: hojas antropológicas, cuadro de resultados, cuenta de ingresos y gastos. Museo Pedagógico Nacional. 1901-1924. Madrid: Imp. Rojas Cosano.

Colonias escolares de vacaciones, Las: hoja antropológica. Museo Pedagógico Nacional. 1907. Madrid: Imp. Rojas.

DEL POZO ANDRÉS, M. ${ }^{a}$ MAR. 1989. "La innovación metodológica y la formación del profesorado en la Escuela de Estudios Superiores del Magisterio", en A. Molero y M. ${ }^{a}$ M. Del Pozo (eds.), Un precedente bistórico en la formación universitaria del profesorado español. Escuela de Estudios Superiores del Magisterio (1909-1932): 65140. Madrid: Dpto. de Educación de la Universidad de Alcalá de Henares.

DíAZ G. VianA, Luis (coord.). 1997. Juego de niños: cantos e imágenes en los procesos de aprendizaje cultural. Oyarzun: Sendoa, 2 vols.

Díaz MAderuelo, RAFAel. 1976. Antropología y educación: Estudio de un caso desde la perspectiva de la Teoría General de Sistemas. Tesis doctoral. Universidad Complutense de Madrid.

Díaz MuÑoz, PEDro. 1911. Antropología y Pedagogia. Valladolid: Imp. y Librería Nacional y Extranjera de Andrés Martín (5..$^{3}$ ed.).

DíAZ DE RADA, ÁNGEL. 1993. La escuela y sus mediaciones. Una etnografía para la crítica de la visión instrumental de la enseñanza. Tesis doctoral. UNED. 
DORFMAN, ARIEL. 2002. Patos, elefantes y béroes. La infancia como subdesarrollo. Madrid: Siglo XXI. Reed. de la 2..$^{\text {a }}$ ed. de 1985.

Exposición del Traje Regional. Guia. 1925. Madrid.

GARCía CASTAÑo, FRANCISCO J. 1989. Transmisión cultural en una institución educativa universitaria. Análisis antropológico de las relaciones docente-discente. Tesis doctoral. Universidad Complutense de Madrid.

- 1994. "Antropología pedagógica" en C. Ortiz y L. A. Sánchez (eds.), Diccionario bistórico de la antropología española: 91-97. Madrid: CSIC.

- y Rafael A. Pulido Moyano. 1994. Antropología de la educación. Madrid: Eudema.

GIL, BONIFACIO. 1964a. Cancionero infantil universal. Madrid: Aguilar.

-. 1964b. Cancionero infantil (antología). Madrid: Taurus.

Giner de los Ríos, Hermenegildo. 1877. Programas de Biología y Antropología. Málaga: Imprenta de la Revista de Andalucía.

GREENWOOD, DAVYDD. 1996. "La etnografía y la etnicidad en España y los Estados Unidos: un replanteamiento de la división del trabajo de las ciencias sociales". Anales de la Real Academia de Ciencias Morales y Políticas XIVIII (73): 383-415.

Herrálz, Gregorio. 1896. Tratado de antropología y pedagogía. Madrid: Viuda de Hernando y Cía.

Hoyos SÁINZ, LUIS DE. 1917. La Antropología. Métodos y problemas. Madrid: Imprenta Clásica Española.

- 1922. "Etnografía española. Cuestionario y bases para el estudio de los trajes regionales". Actas y Memorias de la Sociedad Española de Antropología, Etnografía y Prebistoria I: 91-129.

IANNY, F. A. y E. STORY. 1976. Cultural Relevance and Educational Issues. Readings in Anthropology and Education. Boston: Liltle Brown.

ITARD, JEAN. 1982. Victor del'Aveyron. Madrid: Alianza. Con notas y traducción de Rafael Sánchez Ferlosio.

Juliano, DOlOREs. 1996. "Antropología de la educación”, en Joan Prat y Ángel Martínez (eds.), Ensayos de antropología cultural. Homenaje a Claudio Esteva-Fabregat.: 278285. Barcelona: Ariel.

LARREA PALACÍN, ARCADIO DE. 1958. El folklore y la escuela: ensayo de una didáctica folklórica. Burgos: Imp. Aldecoa.

Lisón TOlOSANA, CARMElO. 1977. "Una gran encuesta en 1901-1902 (Notas para la historia de la antropología social en España)", en Antropología social en España: 105179. Madrid: Akal. $2^{\text {a }}$ ed.

MARTínez TORNER, EDUARDO. 1936. El folklore en la escuela. Madrid: Publicaciones de la Revista de Pedagogía.

MEAd, Margaret. 1972. Educación y cultura. Buenos Aires: Paidós.

- y MARTHA WOLFENSTEIN (eds.). 1955. Childbood in Contemporary Culture. Chicago: The University of Chicago Press.

Molero, ANTONIO y M. a MAR DEL POZO (eds.). 1989. Un precedente bistórico en la formación universitaria del profesorado español. Escuela de Estudios Superiores del Magisterio (1909-1932). Madrid: Dpto. de Educación de la Universidad de Alcalá de Henares.

Nohl, Herman. 1965. Antropología pedagógica. México: FCE. 1. a ed. en alemán, 1938.

OrTiz García, CARMEN. 1987. Luis de Hoyos Sáinz y la antropología española. Madrid: CSIC. 
- 1988a. Historia del pensamiento antropológico en España: Luis de Hoyos Sáinz. Madrid: Universidad Complutense, 2 vols.

- 1988b. "Contribución de la Escuela Superior del Magisterio al estudio del traje regional español (1914-1936)". Revista de Dialectología y Tradiciones Populares. XIIII: 445-457.

- 1996. "La antropología española en el primer tercio del siglo xx: líneas de continuidad y ruptura”, en E. Aguilar (ed.), De la construcción de la bistoria a la práctica de la antropología en España: 121-130. Zaragoza: Instituto Aragonés de Antropología/FAAEE.

Otero Urtaza, Eugenio. 1994. Manuel Bartolomé Cossio: pensamiento pedagógico y acción educativa. Madrid: Centro de Publicaciones del Ministerio de Educación y Ciencia.

Puig-SAmper, Miguel A. y ANDRÉS GAlerA. 1983. La antropología española del siglo XIX. Madrid: CSIC:

RONZÓn, ElENA. 1991. Antropología y antropologías. Ideas para una bistoria crítica de la antropología española. Oviedo: Pentalfa.

SALCEDO Y GINESTAL, ENRIQUE. 1900. Las colonias escolares de vacaciones en España durante los años de 1887 a 1897. Madrid: Imp. de Ricardo Rojas.

SÁNCHEz GómEZ, LuIS A. 1994. "Tubino y Oliva, Francisco María", en C. Ortiz y L. A. Sánchez (eds.), Diccionario bistórico de la antropología española: 666-670. Madrid: CSIC.

SCHEUERL, HANS. 1985. Antropología pedagógica: introducción bistórica. Barcelona: Herder. Tubino, Francisco M. ${ }^{\text {a }}$ 1877. "Recherches d'Anthropologie Social". Extrait de la Revue d'Antbropologie 1.

Velasco, honorio; Francisco J. García CASTAÑo y Ángel Díaz de Rada (eds.). 1993. Lecturas de antropología para educadores. El ámbito de la antropología de la educación y de la etnografía escolar. Madrid: Trotta. 